\title{
BLIND POINT-SOURCE IMAGE RESTORATION USING SUBSPACE TECHNIQUES
}

\author{
Brent A. Chipman and Brian D. Jeffs \\ Department of Electrical and Computer Enginecring, Brigham Young University \\ 459CB, Provo, U'T 84602, bjeffs@ee.byu.edu
}

\begin{abstract}
Blind point-source inage restoration refers to the problem of high resolution recovery of point-like sources which are blurred by an unknown point spread function (psf). Applications include astronomical star field localization, magnctoencephalogram brain current imaging, and seismic deconvolution. This paper shows that with suitable constraints, the problem can be cast in the language of subspace decomposition as used in blind signal copy algorithms for digital wircless communications. Assuming a separable psf, we propose a deterministic, non-iterative ESPRIT-like solution to the restoration problem. We next extend the algorithm to non-separable psf's by approximating them as a series expansion of a few separable components.
\end{abstract}

\section{INTRODUCTION}

In this paper we consider the blind restoration of pointlike source images that have been corrupted by noise and blurred by unknown point spread functions (psf). These problems arise in processing astronomical star field frames, magnetoencephalograms imaging of current dipole distributions of brain neural activity, and other targeting applications where high resolution localization of a few discrete sources is the primary aim. We assume one frame of blurred observation data is available with no knowledge of the blurring psf.

Array processing algorithms, like ESPRIT [1] and MUSIC, are related to this problem because they inherently deal with point sources. Recently the MUSIC algorithm was used to restore point-source images with known blur [2]. In this paper, we propose an ESPRIT-like algorithm for restoration with unknown blur. The method is noniterative, deterministic, and operates on a single image frame with unknown blur. This formulation requires the fairly stringent constraint that the blurring psf be separable along perpendicular axes. This type of blur, however, is not unrealizable. Long exposure atmospheric turbulence blur has been shown to be approximately circularly Gaussian, which is always separable.

An application of particular interest to us is blind restoration of adaptive optics ( $\mathrm{AO})$ telescope images of star fields. The AO system removes much of the atmospheric-turbulenceinduced blurring, but a residual random, unknown blur remains. The general structure of the blur is known to be

This work was supported by the Air Force Office of Scientific Research, grant no. F49620-93-C-0063 99-0828.
Lorentzian [3], which is well approximated by the sum of two Gaussian blurs. With this motivation we study images blurred by non-separable psf's which can be approximated with a finite series expansion sum of separable blurs. A method is introduced for separately estimating the $x$ and $y$ positions of the point sources in the non-separable case.

The assumed model for the input image, a point-like scene with $d$ sources, is

$$
f(x, y)=\sum_{k=1}^{d} a_{k} \delta\left(x-x_{k}\right) \delta\left(y-y_{k}\right) .
$$

The $k^{\text {th }}$ point source has amplitude $a_{k}$ and position $\left(x_{k}, y_{k}\right)$. The observed image is given by

$$
g(x, y)=h(x, y) * f(x, y)+\eta(x, y) .
$$

Here the symbol '*' signifies two dimensional convolution, $h(x, y)$ is the spatial domain psf, and $\eta(x, y)$ is additive noise. The problem at hand is to estimate point-source position pairs, $\left(x_{k}, y_{k}\right)$, and their associated amplitudes.

\section{RESTORATION WITH SEPARABLE BLUR}

For a separable blur, $h(x, y)=h_{x}(x) h_{y}(y)$. Assuming that the output data is available in a sampled representation, $g[m, n]$, the two-dimensional discrete Fourier transform of the output can be written as $g(\xi, \zeta)$, where $\xi$ and $\zeta$ represent the $x$ and $y$ spatial frequency variables respectively. Sampling this 2-D DFT with $N$ spatial frequency samples along each axis, $\xi_{i}=\zeta_{i}=\frac{2 \pi(i-1)}{N}$ with $i=1 \cdots N$, leads to a matrix representation of the frequency domain data: $\mathbf{G}_{i, j}=g\left(\xi_{j}, \zeta_{i}\right)$. Incorporating equations (1) and 2) into this sampled frequency domain model yields the following matrix representation,

$$
\begin{aligned}
\mathbf{G} & =\mathbf{H}_{y} \mathbf{V}(\mathbf{y}) \mathbf{A V}^{T}(\mathbf{x}) \mathbf{H}_{x}^{T}, \text { where } \\
\mathbf{H}_{y} & =\operatorname{Diag}\left\{\left[h_{y}\left(\zeta_{1}\right), \cdots, h_{y}\left(\zeta_{N}\right)\right]^{T}\right\}, \\
\mathbf{V}(\mathbf{y}) & =\left[\mathbf{v}\left(y_{1}\right) \cdots \mathbf{v}\left(y_{d}\right)\right] \\
\mathbf{v}\left(y_{k}\right) & =\left[e^{-j \zeta_{1} y_{k}} \cdots e^{-j \zeta_{N} y_{k}}\right]^{T}, \\
\mathbf{A} & =\operatorname{Diag}\left\{\left[a_{1}, \cdots, a_{d}\right]^{T}\right\} .
\end{aligned}
$$

and where $\operatorname{Diag}\{\mathbf{x}\}$ indicates a diagonal matrix formed from vector $\mathbf{x} . \mathbf{H}_{x}, \mathbf{V}(\mathbf{x})$, and $\mathbf{v}\left(x_{k}\right)$ are defined similarly. Note that the $k^{t h}$ element of $\mathbf{x}, x_{k}$, is linked to corresponding element $y_{k}$ of $\mathbf{y}$ in specifying $x$ and $y$ positions of a single source point. 
Following the development of Swindlehurst and Gunther (which they used for blind resolution of overlapping echoes in digital communications) [4], we compute the singular value decomposition $\mathbf{G}=\mathbf{U S} \mathbf{S}^{H}$, where $\mathbf{U}$ and $\mathbf{V}$ are $N \times d$ and $\mathbf{S}$ is $d \times d$. In the noiseless case, signal subspace matrices are defined as

$$
\mathbf{E}_{y}=\mathbf{U} \mathbf{S}^{1 / 2} \text {, and } \mathbf{E}_{x}=\overline{\mathbf{V}} \mathbf{S}^{1 / 2},
$$

where $\overline{\mathbf{V}}=\operatorname{conj}(\mathbf{V})$. These matrices can be related to the model in the following fashion

$$
\begin{aligned}
& \mathbf{E}_{y}=\mathbf{H}_{y} \mathbf{V}(\mathbf{y}) \mathbf{A}^{1 / 2} \mathbf{T} \\
& \mathbf{E}_{x}=\mathbf{H}_{x} \mathbf{V}(\mathbf{x}) \mathbf{A}^{1 / 2}\left(\mathbf{T}^{T}\right)^{-1},
\end{aligned}
$$

where $\mathbf{T}$ is some unknown full rank matrix. Let us define $\mathrm{V}_{1}(\mathbf{x})$ as the first $N-\delta$ rows of $\mathrm{V}(\mathbf{x})$ and $\mathrm{V}_{2}(\mathrm{x})$ as the last $N-\delta$ rows of $\mathbf{V}(\mathbf{x})$. This is equivalent to selecting two shifted sensor sub-arrays in ESPRIT based direction of arrival estimation. Since the $\mathbf{V}$ matrices are Vandermonde, $\mathbf{V}_{2}(\mathbf{x})=\mathbf{V}_{1}(\mathbf{x}) \Phi_{x}$ where

$$
\Phi_{x}=\operatorname{Diag}\left[1, e^{-j 2 \pi \delta x_{2} / N}, \cdots, e^{-j 2 \pi \delta x_{d} / N}\right] .
$$

Similarly defining 1 and 2 matrices for $\mathbf{H}_{x}, \mathbf{H}_{y}, \mathbf{E}_{x}, \mathbf{E}_{y}$, and $\mathrm{V}(\mathrm{y})$ leads to the following relations:

$$
\begin{aligned}
\mathbf{H}_{y, 1} \mathbf{E}_{y, 2} & =\mathbf{H}_{y, 2} \mathbf{E}_{y, \perp} \Psi_{y}, \\
\Psi_{y} & =\mathbf{T}^{-1} \Phi_{y} \mathbf{T}, \\
\mathbf{H}_{x, 1} \mathbf{E}_{x, 2} & =\mathbf{H}_{x, 2} \mathbf{E}_{x, 1} \Psi_{x}^{T}, \\
\Psi_{x} & =\mathbf{T}^{-1} \Phi_{x} \mathbf{T} .
\end{aligned}
$$

This observation implies two equivalent constraints on the solution. First, $\Psi_{x}$ and $\Psi_{y}$ must have an equivalent set of eigenvectors and second, $\Psi_{x}$ and $\Psi_{y}$ must commute.

\subsection{The Optimal Solution}

An optimal solution to this problem (in the least squares sense) can be defined as the solution to a constrained minimization problem. First, the following definitions are made

$$
\Delta_{y}=\mathbf{H}_{y, 2}{ }^{-1} \mathbf{H}_{y, 1} \text {, and } \Delta_{x}=\mathbf{H}_{x, 2}{ }^{-1} \mathbf{H}_{x, 1} \text {. }
$$

The solution is

$$
\begin{aligned}
& \hat{\Delta}_{y}, \hat{\Delta}_{x}, \hat{\Psi}_{y}, \hat{\Psi}_{x}= \\
& \quad \arg \min _{\Delta_{y, x}, \Psi_{y, x}}\left\|\left[\begin{array}{c}
\Delta_{y} \mathbf{E}_{y, 2} \\
\Delta_{x} \mathbf{E}_{x, 2}
\end{array}\right]-\left[\begin{array}{c}
\mathbf{E}_{y, 1} \Psi_{y} \\
\mathbf{E}_{x, 1} \Psi_{x}^{T}
\end{array}\right]\right\|_{F}^{2}
\end{aligned}
$$

subject to the constraint

$$
\operatorname{eigv}\left\{\Psi_{y}\right\}=\operatorname{eig}\left\{\Psi_{x}\right\}, \text { or } \Psi_{x} \Psi_{y}-\Psi_{y} \Psi_{x}=0,
$$

where $\operatorname{eig}\{A\}$ denotes the matrix of eigenvectors of $A$. Once the $\Psi_{x}$ and $\Psi_{y}$ matrices are known, the position estimates are given by

$$
x_{i}=\frac{N \angle \lambda_{x, i}}{2 \pi \delta}, \quad y_{i}=\frac{N \angle \lambda_{y, i}}{2 \pi \delta}
$$

where $\lambda_{x, i}$ and $\lambda_{y, i}$ are the $i^{t h}$ eigenvalues of $\Psi_{x}$ and $\Psi_{y}$ respectively.

\subsection{A Suboptimal Solution}

Though the solution presented above is optimal, it is difficult to compute the joint minimization of equation (10). The following suboptimal approach performs the minimizations sequentially. Ignoring the constraint, it is easy to solve for $\Psi_{y}$ and $\Delta_{y}[4]$,

$$
\hat{\delta}_{y}=\arg \min _{\delta_{y}} \delta_{y}^{I I}\left[\mathbf{P}_{E_{y, 1}}^{\perp} \odot\left(\mathbf{E}_{y, 2} \mathbf{E}_{y, 2}^{H}\right)^{T}\right] \delta_{y}
$$

where $\delta=\operatorname{diag}(\Delta)$ is the vector extracted from the diagonal of $\Delta, \mathbf{P}$ denotes a projection matrix with respect to the subscript, and $\odot$ denotes a Schur matrix product. Using $\hat{\Delta}=$ Diag $\hat{\delta}$ yields

$$
\hat{\Psi}_{y}=\left(\mathbf{E}_{y, 1}^{H} \mathbf{E}_{y, 1}\right)^{-1} \mathbf{E}_{y, 1}^{H} \hat{\Delta}_{y} \mathbf{E}_{y, 2} .
$$

The estimate for $\mathbf{T}$ is $\hat{\mathbf{T}}=\operatorname{eig}\left\{\hat{\Psi}_{y}\right\}^{-1} . \Delta_{x}$ and $\Phi_{x}$ are then estimated as

$$
\hat{\Delta}_{x}, \hat{\Phi}_{x}=\arg \min _{\Delta_{x}, \Phi_{x}}\left\|\Delta_{x} \mathbf{E}_{x, 2} \hat{\mathbf{T}}^{T}-\mathbf{E}_{x, 1} \hat{\mathbf{T}}^{T} \Phi_{x}\right\|_{F}^{2}
$$

With a little algebra and calculus, it can be shown [5] that equation (15) is equivalent to

$$
\begin{aligned}
\hat{\delta}_{x} & =\mathbf{C}^{-1} \mathbf{D} \phi_{x}, \text { and } \\
\hat{\phi}_{x} & =\arg \min _{\phi_{x}} \phi_{x}^{H}\left(\mathbf{E}-\mathbf{D}^{H} \mathbf{C}^{-1} \mathbf{D}\right) \phi_{x}
\end{aligned}
$$

where $\phi_{x}=\operatorname{diag}\left(\boldsymbol{\Phi}_{x}\right), \delta_{x}=\operatorname{diag}\left(\Delta_{x}\right), \mathbf{E}=\mathbf{I} \odot \mathbf{R}^{H} \mathbf{R}$, $\mathbf{C}=\mathbf{I} \odot\left(\mathbf{Q} \mathbf{Q}^{H}\right)^{T}, \mathbf{D}=\mathbf{R} \odot \overline{\mathbf{Q}}, \mathbf{Q} \stackrel{\text { def }}{=} \mathbf{E}_{x, 2}(\hat{\mathbf{T}})^{T}$, and $\mathbf{R} \stackrel{\text { def }}{=}$ $\mathbf{E}_{x, 1}(\hat{\mathbf{T}})^{T}$. It is easy to solve for $\phi_{x}$ with a singular value decomposition. $x_{i}$ positions are computed directly from $\hat{\phi}_{x}$ using equation (6), and $\hat{\delta}_{x}$ is used to estimate $\mathbf{H}_{x}$. The point source $y$ positions are similarly computed by switching the roles of $x$ and $y$ in the derivation above.

\section{NON-SEPARABLE BLURRING FUNCTIONS}

When $h(x, y)$ is not separable, the above method fails, which limits use of the algorithm to a specific class of images. This section introduces a method that works with nonseparable blurs. Suppose we have a point-source image that is blurred by a psf with exactly $p$ separable components, i.e. $h(x, y)=\sum_{i=1}^{p} h_{y i}(x) h_{x i}(y)$. Note that for sampled images, such a finite series expansion of the blur into separable components is always possible by letting $h_{y i}(m)$ and $h_{x i}(n)$ be the Fourier basis functions. The received image can be modeled as

$$
g(x, y)=\sum_{k=1}^{d} \sum_{i=1}^{p} a_{k} h_{x i}\left(x-x_{k}\right) h_{y i}\left(y-y_{k}\right)
$$

Taking a 1-D DFT of the columns and taking $N$ samples as before yields

$$
\mathbf{G}_{y}=\sum_{i=1}^{p} \mathbf{H}_{y i} \mathbf{V}(\mathbf{y}) \mathbf{A}_{y i}+\mathbf{N}_{y}
$$


Each of the separable components are of rank $d$, so the total rank in the noise free $\mathbf{G}_{y}$ must be $q<p d$. Letting $\mathbf{E}$ be the first $q$ singular vectors from the SVD of $\mathbf{G}_{y}$ leads to

$$
\left[\mathbf{H}_{y 1} \mathbf{V}(\mathbf{y})|\cdots| \mathbf{H}_{y p} \mathbf{V}(\mathbf{y})\right] \in \operatorname{span}\{\mathbf{E}\}
$$

Thus for each $i$ there exists a $q \times d$ matrix $\mathbf{T}_{i}$ such that

$$
\mathbf{E T}_{i}=\mathbf{H}_{y i} \mathbf{V}(\mathbf{y}) \text {. }
$$

This can be recast into the least squares problem

$$
\hat{\mathbf{H}}_{y i}, \hat{\mathbf{T}}_{i}, \hat{\mathbf{y}}=\arg \min _{H, T, y}\left\|\mathbf{H}_{y i} \mathbf{V}(\mathbf{y})-\mathbf{E} \mathbf{T}_{i}\right\|_{F^{\prime}}^{2} .
$$

Using the sub-optimal sequential estimation approach to solve for $\mathbf{T}_{i}$ first gives

$$
\hat{\mathbf{T}}_{i}=\left(\mathbf{E E ^ { H }}\right)^{-1} \mathbf{E}^{I I} \mathbf{H}_{y i} \mathbf{V}(\mathbf{y}) .
$$

Reinserting this solution into equation (21) yields

$$
\hat{\mathbf{H}}_{y i}, \hat{\mathbf{y}}=\arg \min _{I, y}\left\|\mathbf{P}_{E}^{\perp} \mathbf{H}_{y i} \mathbf{V}(\mathrm{y})\right\|_{l^{\prime}}^{2}
$$

which can be expanded in exactly the same manner as the previous section's solution for $\delta$ to obtain

$$
\hat{\mathbf{h}}_{y i}, \hat{\mathbf{y}}=\arg \max _{h, y} \mathbf{h}_{y i}^{H} \Theta(\mathbf{y}) \mathbf{h}_{y i},
$$

where

$$
\Theta(\mathbf{y})=\left(\mathbf{E} \mathbf{E}^{H}\right) \odot\left(\mathbf{V}(\mathbf{y}) \mathbf{V}^{I I}(\mathbf{y})\right)^{T} .
$$

If the vectors $\mathbf{h}_{y i}$ are constrained to be of unit magnitude, the maximum value of the quadratic form in equation (24) will be $\lambda_{\max }$, the maximum eigenvalue of $\Theta(y)$. Thus, regardless of the values of the true $\mathbf{h}_{y i}$ vectors, the solution for positions can be expressed independent of the unknown blur with the following reformulation

$$
\hat{\mathbf{y}}=\arg \max _{y}\{\max \operatorname{cigval}(\Theta(\mathbf{y}))\} .
$$

This appears to be a daunting equation to solve numerically. At first glance it seems as though there is no hope without an exhaustive search over the $d$ dimensional parameter space in $y$, (i.e. compute $\Theta(y)$ for all possible configurations of $d$ points positioned along the $y$ axis, calculating eigenvalues at each step.) It is possible however to solve the problem with at most 2 scans over a one dimensional parameter space by exploiting underlying structure.

While equation (20) holds for the vector $y$ consisting of all $d$ of the $y$ positions for point sources, it also holds for a shorter y containing at least two elements (points). Correspondingly, equation (26) will achieve maxima for any size vector $\mathbf{y}$ as long as all the specified positions match some subset of points in truth image $f(x, y)$. In particular, if only two points are chosen, the largest eigenvalue of $\Theta(y)$ will achieve a local maximum whenever the two $y$ parameters are separated by any distance which exists between some actual point-source pair. We note that in any truly blind restoration, only relative distance between features can be recovered. Absolute position is irrelevant because global shifts with respect to a reference frame can also be interpreted as arising from a non-centralized blur psf.

For example, consider an image with 5 points located at positions $y=\{10,20,25,37,39\}$. The set of all pairwise differences in position is $\{2,5,10,12,14,15,17,19,27,29\}$. Defining a two point position vector as $\mathrm{y}=[0, y]^{T}$, turns equation (26) into the following 1-D optimization problem in $y$

$$
f(y)=\max \operatorname{eigval}(\Theta(\mathbf{y})) .
$$

The plot of $f$ as a function of $y$ is called the difference scan, which will manifest peaks at each legitimate difference. It is possible to reduce the computation by appealing to a lemma found in [4] whereby we can reduce the matrix $\Theta(y)$ to a size of $q \times q$, which is smaller than an $N \times N$.

Armed with the difference scan, we can obtain a solution for the position estimates. The two points that are separated by the maximum difference are uniquely determined. To prove this property, assume there are two sets of point pairs, $\left(y_{1}, y_{2}\right)$ and $\left(y_{3}, y_{4}\right)$, which are separated by the same (maximum) distance, $D_{\max }$. For this condition

$$
y_{2}-y_{1}=D_{\max }, \text { and } y_{4}-y_{3}=D_{\max } .
$$

If $y_{1} \leq y_{3} \leq y_{2}$ then clearly $y_{4}-y_{1}>D_{\max }$ which violates the premise that $D_{\max }$ was the maximum difference. The other possible relative position cases lead to a similar conclusion. Therefore, the local peak in equation (27) corresponding to the largest $y$ value localizes the unique point pair distance $D_{\max }$. With this first pair separation uniquely determined, each other position can be uniquely determined between them. Now, define the single parameter point triple

$$
\mathrm{y}=\left[0, D_{\text {max }}, y\right]^{T},
$$

and the solution spectrum, $f(y)$, will have unique peaks at all correct positions, including 0 and $D_{\text {max }}$, when scanning the single scalar parameter $y$.

\section{RESULTS}

The first example shown in Figure 1 is for the separable blur method. The observed image contains six stars convolved with a circularly symmetric Gaussian blurring function with a standard deviation of 3.5 pixels. Two sets of stars are very much blurred together. The top left frame shows the blurred data with white Gaussian noise added at a level of $40 \mathrm{~dB}$ peak SNR. The top right frame shows the position estimates as asterisks, and the correct positions as diamonds. These results are obtained with the $y$ positions estimated without regard to the constraint of equation (11). The bottom left image shows the converse case, where estimates of the $x$ positions do not include the constraint. Note that position estimates are quite accurate (average error less than two pixels), which is remarkable given the noise level and complete lack of knowledge about the blur, $h(x, y)$, other than that it is separable.

The second example shown in Figure 2 illustrates the non-separable method. The same input image is used, except this time the blur is a non-separable Lorentzian function. 'The top left frame shows the blurred image, while the 
top right shows the input image itself. Note that the this truth image corresponds to a smaller window centered on the blurred points in the observed image, and is thus presented at a lower resolution scale. The bottom two graphs are the solution spectra for the $y$ and $x$ positions respectively. We see that the peaks of the spectra indeed correspond to the actual positions represented in the input image.
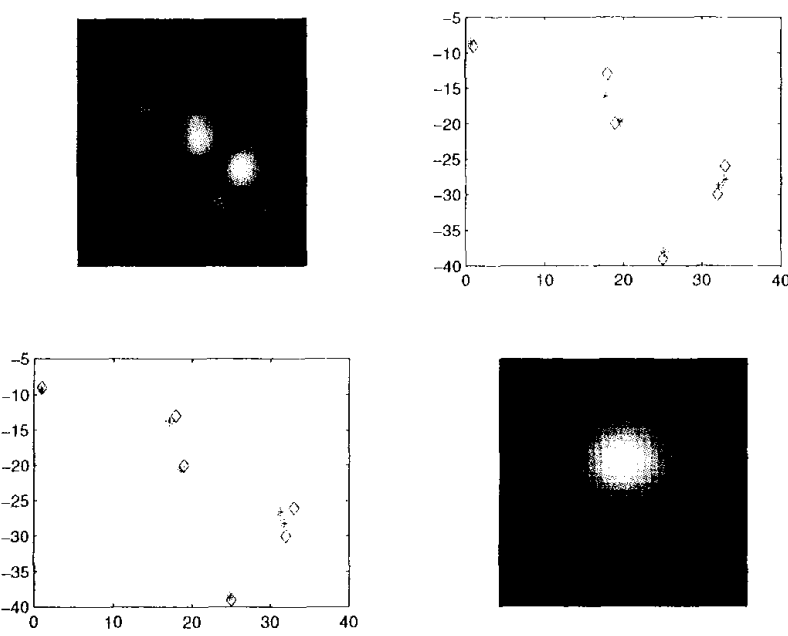

Figure 1: Six star example at $P S N R=40 \mathrm{~dB}$. Top left: Blurred, noisy output. Top right: Position estimates with $y$ fixed. Bottom left: Position estimates with $x$ fixed. Bottom right: Blur estimate.
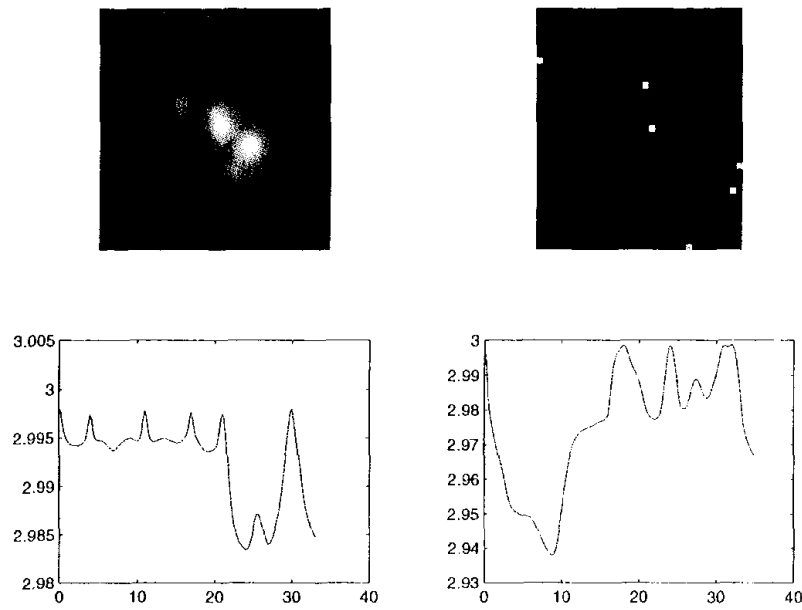

Figure 2: Six star example $200 \mathrm{~dB}$. Top left: Blurred, noisy image. Top right: Truth image. Bottom left: $y$ positions solution spectrum. Bottom right: $x$ position solution spectrum.

\section{CONCLUSIONS}

We have presented two methods of blind point-source image restoration based on deterministic, subspace decomposition algorithms. The underlying point-source image is recovered from a single observation frame without any prior knowledge about the blurring function (in the case of the nonseparable algorithm). If it is known that the blur psf is separable, then an even more computationally efficient algorithm is available. Other known point source restoration methods are iterative, or require knowledge of the blur, or require multiple observation frames with distinct blur, or are based on statistical signal models, or involve lengthy simulated annealing optimization codes $[2,5,6,7]$. We believe the new methods presented here are superior, first in elegance and second in speed. We know of no other deterministic blind method which is optimized for point-source images. The new methods involve extension and adaptation of digital communications algorithms, originally developed for resolving overlapping multipath echoes, so that they can be used in 2-D blind imaging problems. Future work should include further attention to the issue of linking the scparate $x$ and $y$ spectrum scanned position estimates in the non-separable algorithm. Methods of extracting blur psf and point amplitude estimates from the non-separable algorithm are also under study.

\section{REFERENCES}

[1] R. Roy and T. Kailath, "Esprit - estimation of signal parameters via rotational invariance", IEEE Transactions on Acoustics, Speech, and Signal Processing, vol. 37, no. 7, pp. 984-995, July 1989.

[2] Metin Gunsay and Brian D. Jeffs, "Point-source localization in blurred inages by a frequency-domain eigenvector-based method", IEEE Transactions on Image Processing, vol. 4, no. 12, pp. 1602-1612, December 1995.

[3] B. Jeffs, S. Hong, and J. Christou, "Generalized gauss markov model for space objects in blind restoration of adaptive optics telescope images", in Proceedings of the International Conference for Image Processing, $\mathrm{Na}$ gasaki, October 1998, vol. 2, pp. 737-741.

[4] A. Swindlehurst and J. Gunther, "Methods for blind equalization and resolution of overlapping echoes of unknown shape", IEEE Transactions on Signal Processing, vol. 47, no. 5, pp. $1245-1254$, April 1999.

[5] Brent A. Chipman, "Methods of blind point source restoration", Master's thesis, Brigham Young University, 1999

[6] J. Phillips, R. Leahy, and J. Mosher, "Meg-based imaging of focal neuronal current sources", IEEE Transactions on Medical Imaging, vol. 16, no. 3, pp. 338--348, June 1997.

[7] S.M. Jefferies and J.C. Christou, "Restoration of astrononmical images by iterative blind deconvolution", Astrophysical Journal, vol. 415, pp. 862-874, Oct. 1993. 